\title{
Distinct associations of NEDD4L expression with genetic abnormalities and prognosis in acute myeloid leukemia
}

Ming-qiang Chu ${ }^{1,2 \dagger}$, Liu-chao Zhang ${ }^{3 \dagger}$, Qian Yuan ${ }^{1,2}$, Ting-juan Zhang ${ }^{4^{*}}$ and Jing-dong Zhou ${ }^{1 *}$ (D)

\begin{abstract}
Background: There is mounting evidence that demonstrated the association of aberrant NEDD4L expression with diverse human cancers. However, the expression pattern and clinical implication of NEDD4L in acute myeloid leukemia (AML) remains poorly defined.

Methods: We systemically determined NEDD4L expression with its clinical significance in AML by both public data and our research cohort. Moreover, biological functions of NEDD4L in leukemogenesis were further tested by in vitro experiments.

Results: By the public data, we identified that low NEDD4L expression was correlated with AML among diverse human cancers. Expression of NEDD4L was remarkably decreased in AML compared with controls, and was confirmed by our research cohort. Clinically, low expression of NEDD4L was correlated with greatly lower age, higher white blood cells, and higher bone marrow/peripheral blood blasts. Moreover, NEDD4L underexpression was positively correlated with normal karyotype, FLT3 and NPM1 mutations, but negatively associated with complex karyotype and TP53 mutations. Importantly, the association between NEDD4L expression and survival was also discovered in cytogenetically normal AML patients. Finally, a number of 1024 RNAs and 91 microRNAs were identified to be linked to NEDD4L expression in AML. Among the negatively correlated microRNAs, miR-10a was also discovered as a microRNA that may directly target NEDD4L. Further functional studies revealed that NEDD4L exhibited anti-proliferative and pro-apoptotic effects in leukemic cell line K562.

Conclusions: Our findings indicated that NEDD4L underexpression, as a frequent event in AML, was associated with genetic abnormalities and prognosis in AML. Moreover, NEDD4L expression may be involved in leukemogenesis with potential therapeutic target value.
\end{abstract}

Keywords: NEDD4L, Expression, Prognosis, Acute myeloid leukemia

*Correspondence: 1162004751@qq.com; 2111713020@stmail.ujs.edu.cn; zhoujingdong1989@qq.com;2111613022@stmail.ujs.edu.cn

${ }^{\dagger}$ Ming-qiang Chu and Liu-chao Zhang contributed equally to this work

1 Department of Hematology, Affiliated People's Hospital of Jiangsu University, 8 Dianli Rd., Zhenjiang 212002, Jiangsu, People's Republic of China

${ }^{4}$ Department of Oncology, Affiliated People's Hospital of Jiangsu University, 8 Dianli Rd., Zhenjiang 212002, Jiangsu, People's Republic of China

Full list of author information is available at the end of the article

\section{Background}

Acute myeloid leukemia (AML) is a heterogeneous clonal aggressive malignancy characterized by the uncontrolled proliferation and blocked differentiation of myeloid precursor cells [1]. Cytogenetic and genetic abnormalities in leukemic cells lead to a cascade of molecular events, which in turn cause cancer phenotype and inhibit normal hematopoiesis [2]. The genetic alterations emerging in AML has been linked to prognosis and play a crucial role in treatment strategy decision [3]. Moreover, gene 
expression profiling has been widely used in AML, and was also helpful in evaluating the prognostic risk and disease recurrence [4]. At the same time, accumulating studies have reported that high transcript level of $B A A L C$, $M N 1, E R G$, and $W T 1$ was significantly associated with poorer survival in AML [5]. Accordingly, screening and identifying additional AML-related prognostic biomarkers by high-throughput sequencing could precisely recognize higher risk AML, and finally improve the clinical outcome of AML.

The neural precursor cell expressed developmentally downregulated protein 4 (NEDD4) family comprises of nine members including NEDD4, NEDD4-2 (NEDD4L), ITCH, SMURF1, SMURF2, WWP1, WWP2, NEDL1, and $N E D L 2$ in human, which are involved in the regulation of a variety of signaling pathways [6]. NEDD $4 L$ belongs to the evolutionarily conserved NEDD4 family of ubiquitin ligases characterized by a C2 domain, 2-4 WW domains, and a C-terminal HECT-type ubiquitin ligase domain $[7,8] . N E D D 4 L$ is originally discovered in identifying for downregulated genes during the development of the central nervous system $[7,8]$. Recently, there is mounting evidence that showed the association of NEDD $4 L$ expression with prognosis in diverse human cancers [9-16].

Herein, as far as we known, it was the first time to report low expression of NEDD4L in AML. We identified and verified that $N E D D 4 L$ was decreased in AML, and $N E D D 4 L$ underexpression was correlated with specific cytogenetic/genetic abnormalities of AML. Moreover, low expression of $N E D D 4 L$ was associated with clinical outcome in cytogenetically normal AML (CN-AML). Finally, a number of 1024 mRNAs and 91 microRNAs were identified to be linked to NEDD $4 L$ expression in AML. Among the negatively correlated microRNAs, $m i R-10 a$ was also discovered as a microRNA that may directly target $N E D D 4 L$. Further functional studies revealed that $N E D D 4 L$ exhibited anti-proliferative and pro-apoptotic effects in leukemic cell line K562.

\section{Materials and methods \\ CCLE}

The CCLE (Cancer Cell Line Encyclopedia) database (https://www.broadinstitute.org/ccle) focuses on the gene expression, methylation, and mutation data for over 1100 types of cancer cell lines [17]. NEDD4L expression in cancer cell lines was firstly identified by CCLE.

\section{HPA}

The HPA (Human Protein Atlas) database (https://www. proteinatlas.org/) focuses on proteins expression in cells, tissues, and organs [18]. NEDD4L expression in cancer cell lines was further identified by HPA.

\section{GEPIA}

The GEPIA (Gene Expression Profiling Interactive Analysis) database (http://gepia.cancer-pku.cn/) focuses on analyzing the RNA sequencing expression data of 9736 tumors and 8587 normal samples from the TCGA (The Cancer Genome Atlas) and the GTEx (Genotype-Tissue Expression) projects, using a standard processing pipeline [19]. NEDD4L expression in 33 types of cancer patients including AML and controls was analyzed by GEPIA.

\section{BloodSpot}

The Bloodspot (http://servers.binf.ku.dk/bloodspot/) provides a plot of gene expression in hematopoietic cells at different maturation stages based on curated microarray data [20]. NEDD4L expression between among AML subtypes and controls was identified by Bloodspot.

\section{TCGA databases}

TCGA is a landmark cancer genomics program, which molecularly characterized over 20,000 primary cancers and normal samples spanning 33 cancer types. The current study included a total of 173 AML patients with RNA-sequencing data (RNA Seq V2 RSEM) from the databases of TCGA (AML NEJM 2013) downloaded by cBioportal (http://www.cbioportal.org/) [21]. Expression and mutation data of these patients were also obtained by mRNA- and DNA-sequencing. Clinical features and treatment regimens for these patients were as reported [21].

\section{GEO databases}

Gene Expression Omnibus (GEO) is a public functional genomics data repository supporting MIAME-compliant data submissions. Three GEO datasets (GSE12417, GSE6891 and GSE10358) were used to evaluate the prognostic value of NEDD4L expression in AML. Firstly, the effect of $N E D D 4 L$ expression on survival was analyzed in GSE12417 dataset which included 78 and $162 \mathrm{CN}$-AML patients through the online tool Genomicscape (http://genomicscape.com/microarray/ survival.php) [22, 23]. Then, GSE6891 dataset consisted of 187 CN-AML patients as well as GSE10358 dataset comprised of $131 \mathrm{CN}$-AML patients were further used for validation.

\section{Patients and samples}

The validation cohort of 44 AML patients at newly diagnosis time (ND-AML, used ad cases) and 47 AML patients at complete remission (CR) time (CR-AML, used as controls) was also enrolled in this study. The 
detailed information of $44 \mathrm{ND}-\mathrm{AML}$ patients was given in Additional file 1: Table S1. The age and sex between AML and controls presented no significant differences $(P>0.05)$. Bone marrow $(\mathrm{BM})$ samples were collected from these patients. BM mononuclear cells (BMMNCs) separated from BM of these AML patients was used in this study. The current study protocol was approved by the Institutional Ethics Committee of The Affiliated People's Hospital of Jiangsu University, and all the participants provided written informed consents.

\section{RNA isolation and reverse transcription}

Total RNA was isolated form BMMNCs by using Trizol reagent (Invitrogen, Carlsbad, CA) as our pervious literature [24-26]. Reverse transcription was performed as reported [24-26]. The conditions performed as follows: $37^{\circ} \mathrm{C}$ for $15 \mathrm{~min}, 85^{\circ} \mathrm{C}$ for $5 \mathrm{~s}$.

\section{RT-qPCR}

RT-qPCR (real-time quantitative PCR) analysis was performed to detect NEDD4L, CASP3 and CASP8 mRNA using AceQ qPCR SYBR Green Master Mix (Vazyme Biotech Co., Piscataway, NJ). The primers used for NEDD4L expression were 5'-CCCAATAGGTTTGAA ATGAA-3' (forward) and $5^{\prime}$-TAGTTGTCCGTGGCA GAGTA-3' (reverse), primers for CASP3 expression were 5'-AATGGACCTGTTGACCT-3' (forward) and 5'-CTG TTGCCACCTTTCG-3' (reverse), and primers for CASP8 expression were $5^{\prime}$-GAGCCAGGGTGGTTAT- $3^{\prime}$ (forward) and 5'-CCTTTGCGGAATGTAG-3' (reverse). Moreover, $A B L 1$ (housekeeping gene) expression was also detected with the primers $5^{\prime}$-TCCTCCAGCTGT TATCTGGAAGA- $3^{\prime}$ (forward) and $5^{\prime}$-TCCAACGAG CGGCTTCAC- $3^{\prime}$ (reverse). Relative target gene expression was calculated based on the $2^{\Delta \mathrm{CT} \text { target gene (control-sam- }}$ ple) $\div 2^{\triangle C T A B L 1 \text { (control-sample) }}\left(2^{-\Delta \Delta C t}\right)$ formula.

\section{Bioinformatics analysis}

Analysis of differentially expressed genes (DEGs) and microRNAs associated with NEDD4L in AML, and the microRNAs-mRNAs network predictions could refer to our previous study [27].

\section{Cell line and cell culture}

Human leukemic cell lines HEL, HL60, K562, MOLM13, MV4-11, NB4, OCI, SHI-1, SKM-1, THP-1 and U937 as well as human bone marrow stromal cell line HS-5 was cultured in RPMI 1640 medium (BOSTER, Wuhan, China) containing $10 \%$ fetal calf serum (ExCell Bio, Shanghai, China) and grown at $37^{\circ} \mathrm{C}$ in $5 \% \mathrm{CO}_{2}$ humidified atmosphere.

\section{SiRNA transfection}

Knockdown of NEDD4L expression used for lossof-function experiments was done by siRNA. The siNEDD4L (sense strand: 5'-CCUCUGUAAUGAGGA UCAUUU- ${ }^{\prime}$ and antisense strand: $5^{\prime}$-AAAUGAUCC UCAUUACAGAGG-3') [28] were purchased from GenePharma (Shanghai, China). SiRNA transfection was performed using the X-tremeGENE siRNA Transfection Reagent (Roche, Basel, Switzerland) according to the manufacturer's instructions. Transfected cells were used for experiments in $48 \mathrm{~h}$ after siRNA transfection.

\section{Cell proliferation assays}

The tested cells $\left(1 \times 10^{5}\right.$ cells $\left./ \mathrm{mL}\right)$ for $2 \mathrm{~mL}$ per well were seeded in a 6 -well plate. After culturing for 0,1 and 2 days, cells were counted in counting board for three times, respectively.

\section{Cell apoptosis assays}

The tested cells $\left(2 \times 10^{5}\right.$ cells $\left./ \mathrm{ml}\right)$ for $2 \mathrm{ml}$ per well were seeded in a 6-well plate. After culturing for 2 days, cells were used for apoptosis assays which were performed using Annexin V PE Apop Dtec Kit (BD Pharmingen, San Diego, CA) via flow cytometry. Each experiment was repeated three times.

\section{Statistical analysis}

Statistical analysis was accomplished by SPSS 22.0 software package. Pearson's $\chi^{2} /$ Fisher's exact test and Mann-Whitney's U/Kruskal-Wallis $H$ test were used for the comparison of categorical and continuous variables, respectively. The impact of $N E D D 4 L$ expression on leukemia-free survival (LFS)/event-free survival (EFS) and overall survival (OS) was analyzed using the Kaplan-Meier method. The receiver operating characteristic (ROC) curve and area under the ROC curve (AUC) value were applied to determined NEDD4L expression in distinguishing AML from controls. The statistical $P$-values were two-sided and less than 0.05 in all analyses were considered as statistically significant differences.

\section{Results}

\section{Low NEDD4L expression associated with AML}

To investigate $N E D D 4 L$ expression pattern in human cancers, we first used the CCLE databases. It was showed that NEDD4L was the lowest expression level in AML cell lines among 40 types of human cancer cell lines (Fig. 1a). Moreover, low NEDD $4 L$ expression was also closely correlated with myeloid cell lines, which was revealed by the HPA databases (Fig. 1b). Then, we 


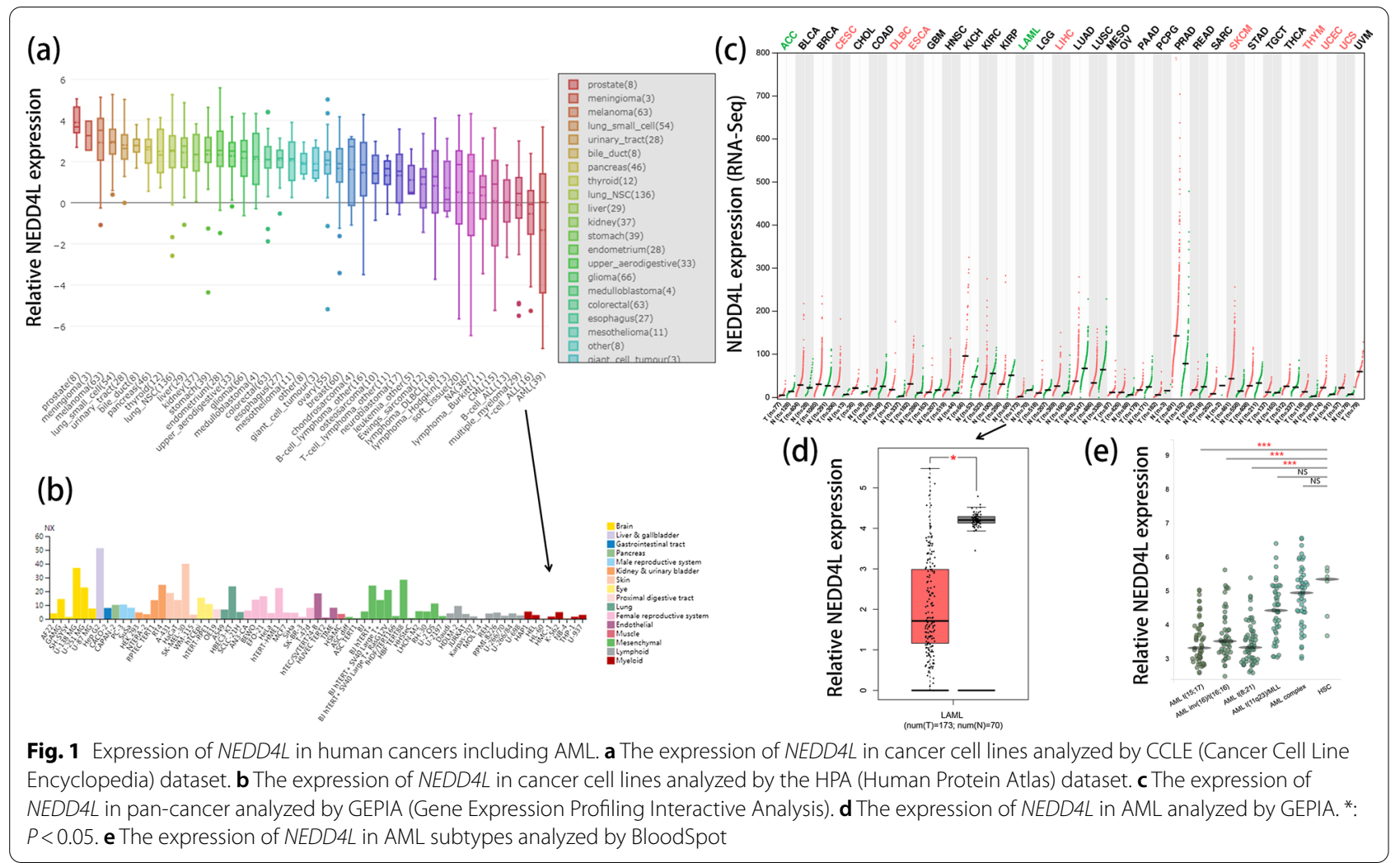

further explored $N E D D 4 L$ expression in human cancer samples and normal controls by using the GEPIA databases. Among the 33 types of human cancers, significant differences of NEDD $4 L$ expression between patients and controls were observed in 10 kinds of human cancers. In detail, eight of them showed increased expression, whereas two of them presented decreased expression including AML (Fig. 1c, d). Moreover, reduced expression of NEDD4L in AML subtypes was also showed by BloodSpot online tool (Fig. 1e). In summary, low NEDD4L expression was closely associated with AML among the 40 types of human cancers.

\section{Validation of NEDD4L expression in AML}

To validate the expression pattern of NEDD4L expression in AML, we further detected NEDD4L mRNA expression in BMMNCs samples of another independent cohort of AML patients who were treated in our hospital. As expectedly, NEDD4L expression was significantly reduced in ND-AML (median 0.073, range 0.000 0.735) compared with CR-AML (median 0.140, range 0.003-1.000) ( $P=0.017$, Fig. 2a). Moreover, ROC analysis revealed that $N E D D 4 L$ expression may be served as a potential biomarker for distinguishing ND-AML from CR-AML with an AUC value of 0.645 (95\% confidence interval: $0.532-0.758, P=0.017$, Fig. $2 b)$. These results further confirmed the low expression pattern of $N E D D 4 L$ in AML and revealed that NEDD4L expression might serve as an underlying biological marker helpful for the diagnosis of AML.

\section{Distinct association of NEDD4L expression with clinical features in AML}

When analyzed the clinical implication of NEDD4L expression in AML, the whole-cohort cases were divided into two groups by the median level of NEDD $4 L$ expression. Comparison of clinic-pathologic characteristics between the two groups was presented in Table 1. AML cases with low NEDD4L expression exhibited markedly lower white blood cell (WBC) counts than those with high $N E D D 4 L$ expression $(P<0.001)$. Moreover, $N E D D 4 L$ low-expressed patients presented quite higher $\mathrm{BM}$ and peripheral blood (PB) blasts than NEDD4L highexpressed patients $(P=0.002$ and 0.005 , receptively). Moreover, significantly differences were found in the distribution of cytogenetics between low and high NEDD $4 L$ expressed groups $(P<0.001)$. Low $N E D D 4 L$ expression was appreciably associated with normal karyotype $(P=0.001)$, hardly correlated with complex karyotypes $(P=0.001$, respectively). To further exhibit the associations of NEDD4L expression with cytogenetic classifications, NEDD $4 L$ expression level among different 

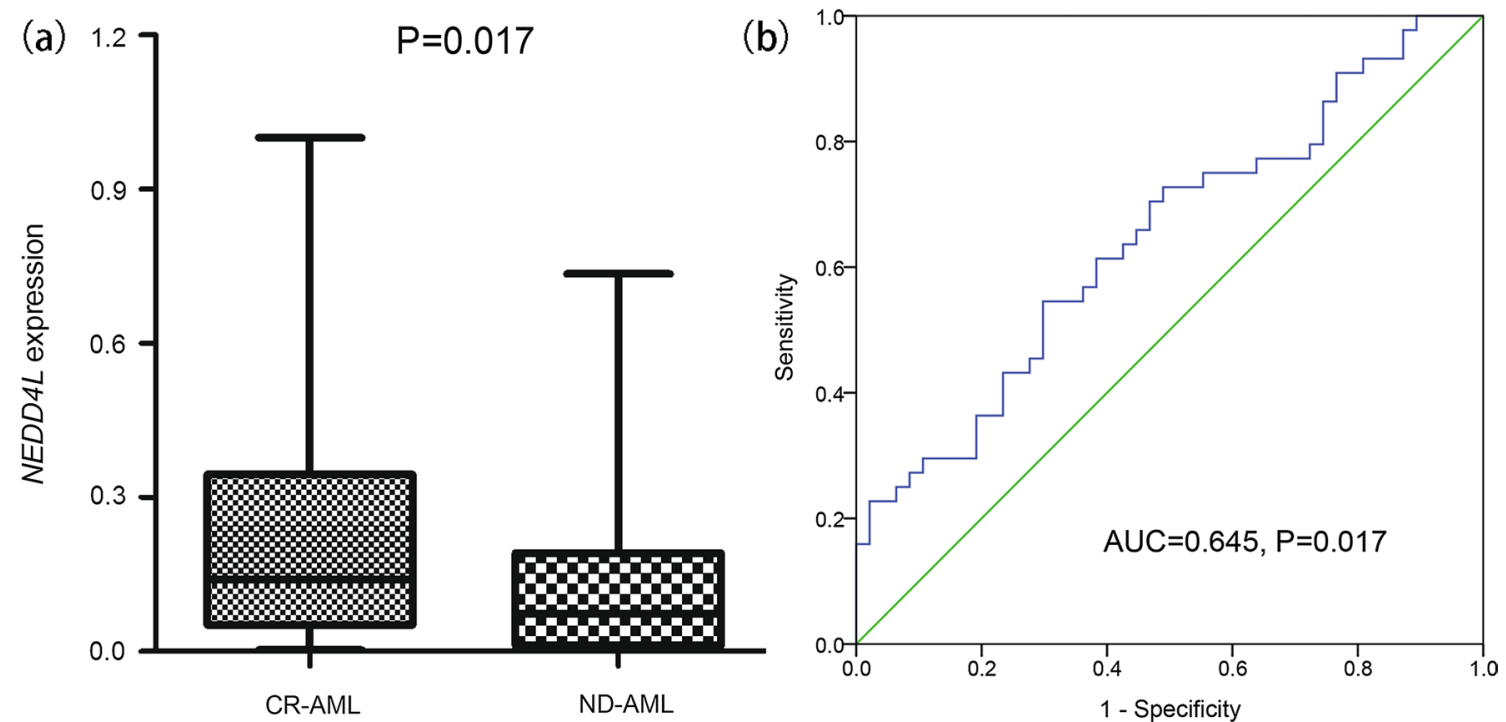

Fig. 2 Validation of NEDD4L expression in AML. a The relative expression of NEDD4L in newly diagnosed AML (ND-AML) and AML achieved CR (CR-AML). Relative NEDD4L expression was calculated based on the $2^{\triangle C T \text { NEDD } 4 L \text { (control-sample) }} \div 2^{\triangle C T \text { ABLI (control-sample) }}\left(2^{-\triangle \triangle C t}\right.$ ) formula. The difference between two groups was compared by Mann-Whitney's $U$ test. b ROC curve analysis of NEDD4L expression in distinguishing ND-AML from CR-AML

karyotypes was further compared $(P<0.001$, Fig. 3a). We further determined the significant associations of NEDD4L expression with common genetic mutations (Table 1). AML patients with low NEDD4L expression showed relatively higher incidence of FLT3, NPM1, and DNMT3A mutations $(P=0.007,0.001$, and 0.051 respectively) but lower frequency of TP53, TET2, and U2AF1 mutations $(P=0.005,0.063$, and 0.064 , respectively) than those with high NEDD4L expression. Moreover, the level of $N E D D 4 L$ expression between the mutant and wild-type groups of FLT3 $(P<0.001)$, NPM1 $(P<0.001)$, DNMT3A $(P=0.033)$, TET2 $(P=0.088)$, TP53 $(P<0.001)$, and U2AF1 $(P=0.033)$ genes was further exhibited (Fig. 3b-g). All these results suggested that aberrant NEDD4L expression was correlated with diverse genetic events in AML.

\section{Prognostic value of NEDD4L expression in AML}

We first determined the effect of $N E D D 4 L$ expression on survival (OS and LFS) in AML from TCGA cohort. Although no remarkably differences of OS and LFS were observed between low- and high- NEDD4L expression groups among total AML $(P=0.952$ and 0.972 , respectively, Additional file 2: Fig. S1), patients with low NEDD4L expression tended to have shorter OS and LFS time than those with high $N E D D 4 L$ expression among CN-AML $(P=0.161$ and 0.122 , respectively, Additional file 2: Fig. S1). Next, we analyzed the GEO datasets (GSE12417) including two cohorts of 78 and $162 \mathrm{CN}$ AML patients to evaluate the prognostic significance of NEDD4L expression in AML. The Genomicscape online tool through Kaplan-Meier analysis demonstrated that low NEDD4L expression was greatly correlated with shorter OS time in both $78 \mathrm{CN}$-AML (probe 212445_s_at: $P=0.033$ and probe 241396 at: $P=0.087$ ) and $162 \mathrm{CN}$-AML (probe 212445_s_at: $P=0.0025$ and probe 241396_at: $P=0.041$ ) cohorts (Fig. 4a). Moreover, the prognostic value of NEDD4L expression on EFS and OS was further confirmed in another two independent cohorts of CN-AML from GSE6891 (probe 212445_s_ at: $P=0.019$ and 0.005 , respectively; probe 241396 at: $P<0.001$ and 0.001 , respectively) and GSE10358 (probe 212445_s_at: $P=0.316$ and 0.076 , respectively; probe 241396_at: $P=0.005$ and 0.001 , respectively) datasets (Fig. 4b, c). Taken together, low NEDD4L expression was correlated with unfavorable prognosis in CN-AML, and might serve as an underlying marker in AML prognosis prediction.

Biological insights of aberrant NEDD4L expression in AML In order to take better understanding of biological insights correlated with aberrant NEDD $4 L$ expression in AML among TCGA databases, we first compared the transcriptomes between high and low NEDD4L expression groups in AML from TCGA cohorts. A number of 1024 DEGs including 933 upregulated and 91 downregulated (high vs low) were obtained between two groups ( $|\log 2 \mathrm{FC}|>1.5, \mathrm{FDR}<0.05$ and $P<0.05$ ) (Fig. 5a, b and Additional file 3: Table S2). The top 50 upregulated genes including $\mathrm{CDH} 1$ and the top 50 downregulated genes 
Table 1 Correlation of NEDD4L expression with clinic-pathologic characteristics in AML

\begin{tabular}{|c|c|c|c|}
\hline \multirow[t]{2}{*}{ Patient's parameters } & \multicolumn{3}{|c|}{ NEDD4L expression } \\
\hline & Low $(n=87)$ & High $(n=86)$ & $P$ value \\
\hline Sex, male/female & $48 / 39$ & $44 / 42$ & 0.353 \\
\hline Median age, years (range) & $55(21-77)$ & $61(18-88)$ & 0.017 \\
\hline Median WBC, $\times 10^{9} / L$ (range) & $31.5(0.9-223.8)$ & $8.6(0.4-297.4)$ & 0.000 \\
\hline Median PB blasts, \% (range) & $50(0-97)$ & $22(0-98)$ & 0.002 \\
\hline Median BM blasts, \% (range) & $76(32-100)$ & $62.5(30-99)$ & 0.005 \\
\hline FAB classifications & & & 0.124 \\
\hline MO & 7 & 9 & 0.611 \\
\hline M1 & 21 & 23 & 0.729 \\
\hline M2 & 22 & 16 & 0.359 \\
\hline M3 & 11 & 5 & 0.188 \\
\hline M4 & 13 & 21 & 0.130 \\
\hline M5 & 12 & 6 & 0.212 \\
\hline M6 & 0 & 2 & 0.246 \\
\hline M7 & 0 & 3 & 0.121 \\
\hline No data & 1 & 0 & 1.000 \\
\hline Cytogenetics & & & 0.000 \\
\hline Normal & 51 & 29 & 0.001 \\
\hline $\mathrm{t}(15 ; 17)$ & 10 & 5 & 0.280 \\
\hline$t(8 ; 21)$ & 6 & 1 & 0.117 \\
\hline Inv (16) & 3 & 7 & 0.211 \\
\hline+8 & 3 & 5 & 0.496 \\
\hline Del (5) & 0 & 1 & 1.000 \\
\hline$-7 /$ del (7) & 2 & 5 & 0.278 \\
\hline $11 q 23$ & 2 & 1 & 1.000 \\
\hline Others & 3 & 11 & 0.028 \\
\hline Complex & 5 & 20 & 0.001 \\
\hline No data & 2 & 1 & 1.000 \\
\hline \multicolumn{4}{|l|}{ Gene mutation } \\
\hline FLT3 $( \pm)$ & $33 / 54$ & $16 / 70$ & 0.007 \\
\hline $\operatorname{NPM1}( \pm)$ & $34 / 53$ & $14 / 72$ & 0.001 \\
\hline DNMT3A $( \pm)$ & $27 / 60$ & $15 / 71$ & 0.051 \\
\hline $\mathrm{IDH} 2( \pm)$ & $9 / 78$ & $8 / 78$ & 1.000 \\
\hline $\mathrm{IDH} 1( \pm)$ & $8 / 79$ & $8 / 78$ & 1.000 \\
\hline TET2 $( \pm)$ & $4 / 83$ & $11 / 75$ & 0.063 \\
\hline RUNX1 ( \pm ) & $10 / 77$ & $14 / 72$ & 0.388 \\
\hline TP53 ( \pm$)$ & $2 / 85$ & $12 / 74$ & 0.005 \\
\hline $\operatorname{NRAS}( \pm)$ & $5 / 82$ & $7 / 79$ & 0.566 \\
\hline CEBPA $( \pm)$ & $7 / 80$ & $6 / 80$ & 1.000 \\
\hline$W T 1( \pm)$ & $7 / 80$ & $3 / 83$ & 0.329 \\
\hline PTPN11 ( \pm ) & $4 / 83$ & $4 / 82$ & 1.000 \\
\hline$K I T( \pm)$ & $3 / 84$ & $4 / 82$ & 0.720 \\
\hline U2AF1 ( \pm$)$ & $1 / 86$ & $6 / 80$ & 0.064 \\
\hline $\operatorname{KRAS}( \pm)$ & $4 / 83$ & $3 / 83$ & 1.000 \\
\hline
\end{tabular}

$A M L$ acute myeloid leukemia, $W B C$ white blood cells, $P B$ peripheral blood, $B M$ bone marrow, FAB French-American-British such as $H 19$ were significantly associated with prognosis in AML by our previous studies $[29,30]$. In addition, the GO (Gene Ontology) analysis demonstrated that these DEGs involved in biologic processes, including multicellular organismal process, system development, multicellular organism development, and biological adhesion (Fig. 5c). Taken together, all the results supported the prognostic impact of low NEDD $4 L$ expression with potential role in AML.

We next determined the microRNA expression signature between low and high NEDD4L expression groups in AML among TCGA databases. We identified 39 differential expressed microRNAs including 27 upregulated and 12 downregulated between two groups (|log2 $\mathrm{FC} \mid>1.0, \mathrm{FDR}<0.05$ and $P<0.05$ ) (Fig. 5d, Additional file 3: Table S2). Downregulated microRNAs such as miR-375, miR-10a, and miR-100 were observed to be overexpressed in AML or have proto-leukemia effects in previous investigations [31-36]. These results together supported the anti-leukemia role and the prognostic effects of NEDD $4 L$ during leukemogenesis. Moreover, among these downregulated microRNAs, miR-10a was also discovered as a microRNA that could directly target NEDD4L (Fig. 5e, Additional file 4: Table S3), which indicated that $N E D D 4 L$ may be seen as a directly target of $m i R-10 a$ in AML.

\section{Validation of the biological role of NEDD4L in AML}

To validate the potential role of NEDD4L in AML development, we next performed in vitro experiments in leukemic cells. Since it is difficult to successfully transfect NEDD4L that has too long coding sequence (CDS $>2000$ bp) into suspension cells, we conducted loss-of-function assays in the highest NEDD4L-expressed cells K562 (Fig. 6A). The successfully knockdown of NEDD4L expression in K562 cells by siRNAs was confirmed through RQ-PCR (Fig. 6B). Expectedly, K562-siNEDD4L cells presented markedly increased proliferation rate (Fig. $6 \mathrm{C}$ ) and decreased apoptosis rate as compared with K562-siNC cells (Fig. 6D-F). Moreover, apoptosis-related markers $C A S P 3$ and $C A S P 8$ were remarkably reduced after $N E D D 4 L$ knockdown in $\mathrm{K} 562$ cells (Fig. 6G and H). All these results together suggested that $N E D D 4 L$ may play a tumor suppressive role in AML biology.

\section{Discussion}

In the current investigation, we for the first time explored NEDD4L expression in AML, and demonstrated that low NEDD4L expression was a frequent event in AML. 


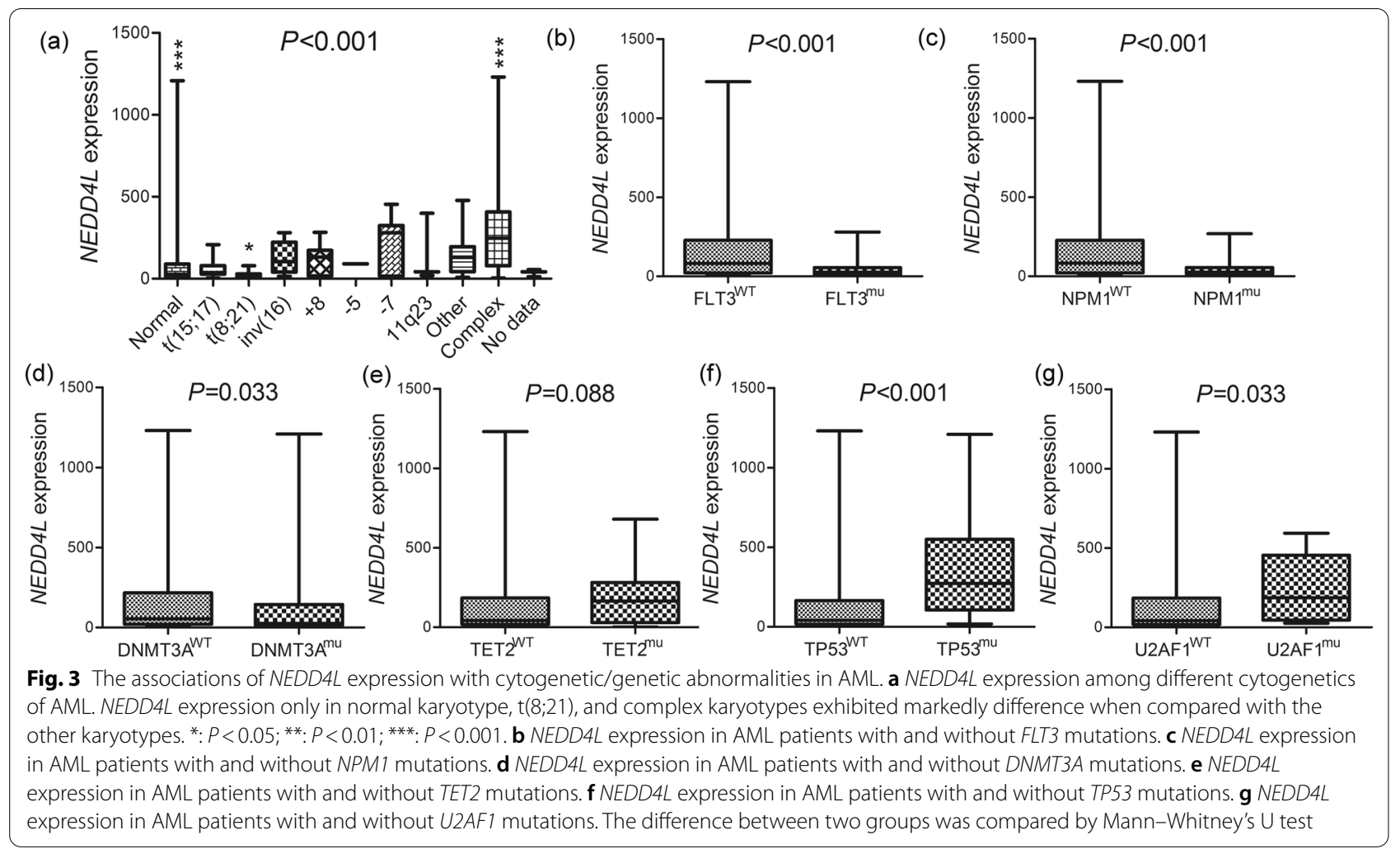

Moreover, NEDD4L expression was appreciably link to the clinical outcome of CN-AML. Although it is the first report regarding the prognostic significance of NEDD $4 L$ expression in AML, several studies have shown the great correlations of $N E D D 4 L$ expression with clinical outcome in solid tumors [9-16]. Reduced expression of NEDD4L correlated with adverse prognosis in non-small cell lung cancer, gastric cancer, hepatocellular carcinoma, ovarian cancer, and malignant glioma [9-16]. In addition, we also determined the potential role of NEDD $4 L$ in AML by further functional study validation, and showed the antiproliferative and pro-apoptotic effects of NEDD $4 L$ in leukemic cell line K562, which suggested that NEDD4L may play a tumor suppressive role in AML biology. However, only a few studies determined the direct role of NEDD $4 L$ in tumorigenesis [10]. Accordingly, further clinical and functional studies are required to explore the potential role of NEDD4L in AML occurrence and development.

Additionally, we also observed a markedly correlation of NEDD4L expression with cytogenetic/genetic classifications in AML by our studies. Underexpression of NEDD4L was observed to be correlated with normal karyotype, FLT3 and NPM1 mutations, but negatively associated with complex karyotype and TP53 mutations. Notably, a recent study also showed that abnormal
NEDD9 expression, a member of NEDD family, was highly correlated with specific French-American-British (FAB) subtypes and karyotypes as well as genetic mutations, which was similar to our results [37]. These results together disclosed that $N E D D 4 L$ underexpression play a key role in CN-AML biology caused by genetic mutations. Future studies are needed to determine the potential associations of aberrant $N E D D 4 L$ expression with genetic abnormalities in CN-AML.

Accumulating studies have reported the expression of $N E D D 4 L$ was regulated by microRNAs during biological process including cancer development. For instance, miR-98 by directly targeting $N E D D 4 L$ played a key role in alleviating renal fibrosis in diabetic nephropathy [38]. MiR-494 inhibited the TGF-beta1/Smads signaling pathway and prevented the development of hypospadias through targeting NEDD4L [39]. Chen et al. demonstrated that IGF-1-enhanced miR-513a-5p signaling desensitized glioma cells to temozolomide through targeting the $N E D D 4 L$-inhibited Wnt/beta-catenin pathway [40]. The $m i R-106 b-25$ cluster through the direct repression of $N E D D 4 L$ mediated breast tumor initiation by the activation of NOTCH1 signaling [41]. Moreover, Zhu et al. reported that the E3 ubiquitin ligase $N E D D 4 / N E D D 4 L$ was directly regulated by $m i R-1$ [42]. 


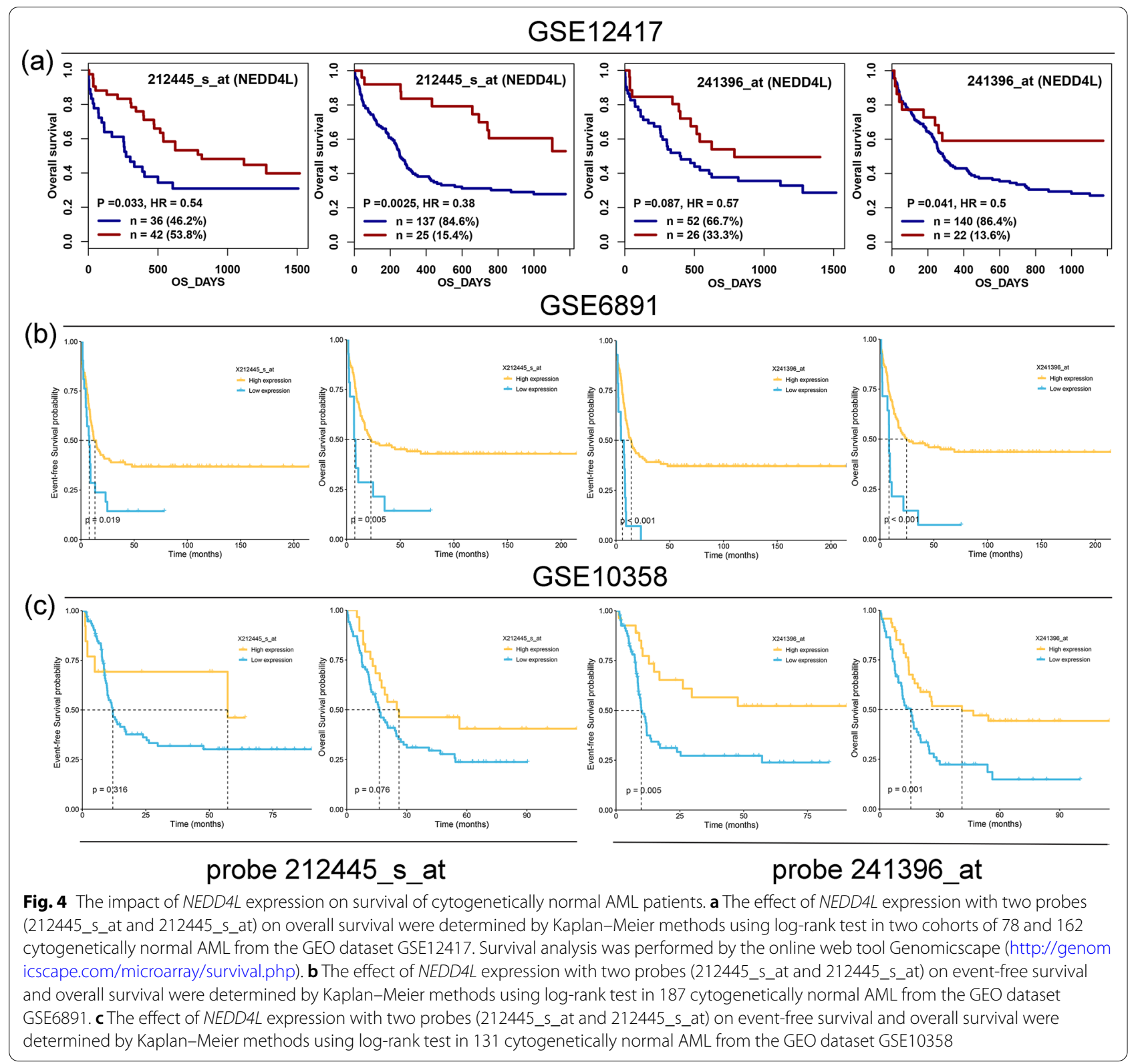

In this study, as far as we know, it is the first time to report the negative correlation of NEDD4L expression with $m i R-10 a$ in AML. Although luciferase assays were not conducted to verify the direct link between $m i R$ $10 a$ and $N E D D 4 L$, an increasingly number of studies revealed the oncogenic role of $m i R-10 a$ with prognostic value in AML [32-34]. All the literatures in turn supported the association of NEDD4L with miR-10a together with prognostic value in AML.

\section{Conclusions}

In summary, our findings demonstrated that NEDD4L underexpression, as a frequent event in AML, was associated with genetic abnormalities and prognosis in AML. Moreover, NEDD4L expression may be involved in leukemogenesis with potential therapeutic target value. 







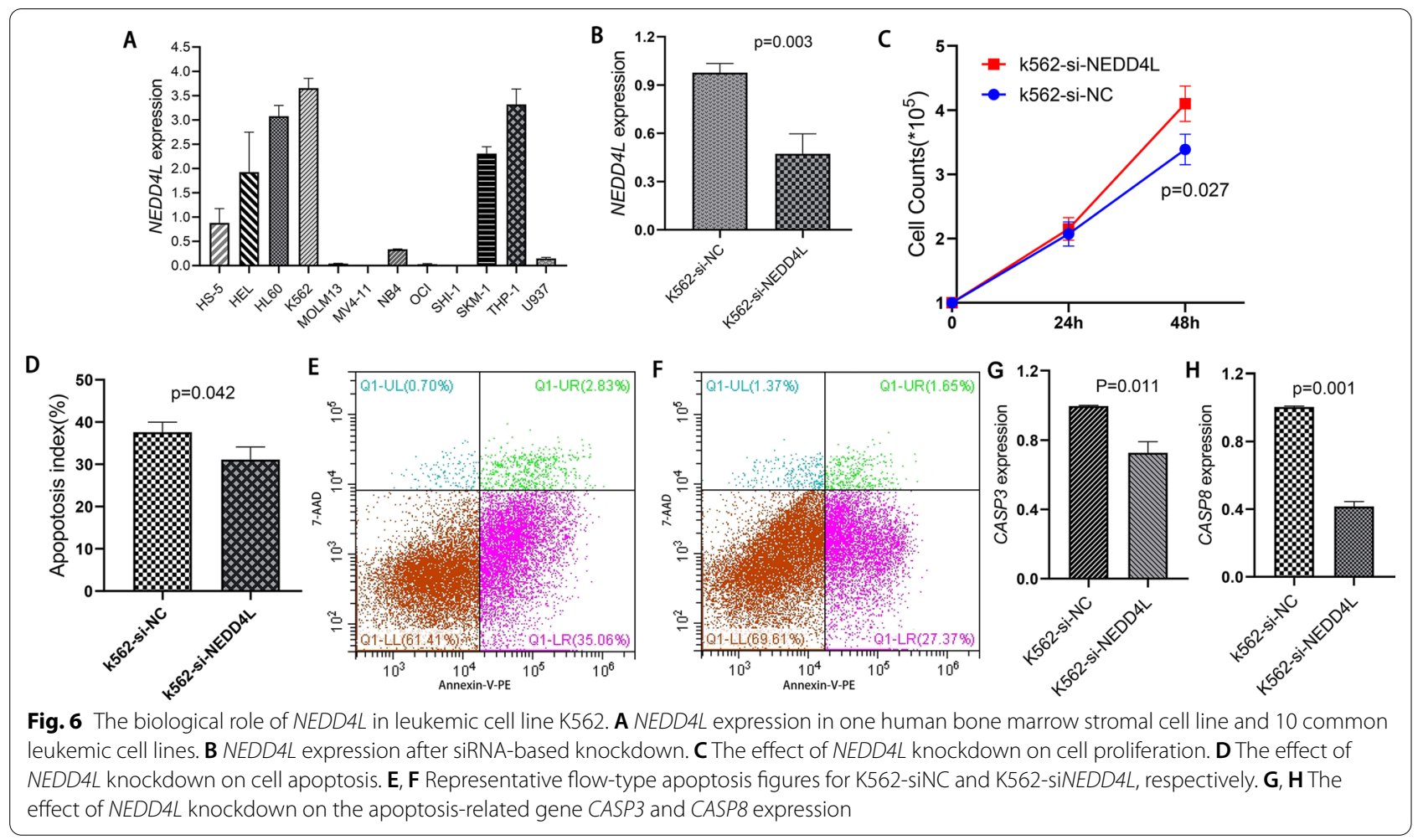

\section{Abbreviations}

AML: Acute myeloid leukemia; NEDD4: Neural precursor cell expressed developmentally downregulated protein 4; FAB: French-American-British; CCLE: Cancer Cell Line Encyclopedia; HPA: Human Protein Atlas; GEPIA: Gene Expression Profiling Interactive Analysis; TCGA: The Cancer Genome Atlas; GTEx: Genotype-tissue expression; CN-AML: Cytogenetically normal AML; GEO: Gene Expression Omnibus; ND-AML: AML at newly diagnosis time; CR: Complete remission; $\mathrm{CR}-\mathrm{AML}$ : $\mathrm{AML}$ at complete remission time; $\mathrm{BM}$ : Bone marrow; BMMNCs: BM mononuclear cells; RT-qPCR: Real-time quantitative PCR; DEGs: Differential expression genes; LFS: Leukemia-free survival; EFS: Event-free survival; OS: Overall survival; ROC: Receiver operating characteristic; AUC: Area under the ROC curve; WBC: White blood cell; GO: Gene ontology; CDS: Coding sequence.

\section{Supplementary Information}

The online version contains supplementary material available at https://doi. org/10.1186/s12935-021-02327-7.

Additional file 1: Table S1. Clinic-pathologic characteristics of AML in our research cohort.

Additional file 2: Figure S1. The impact of NEDD4L expression on survival of AML patients from TCGA cohort. The effects of NEDD4L expression on leukemia-free survival and overall survival were determined by Kaplan-Meier methods using log-rank test in both total AML and CN-AML patients.

Additional file 3: Table S2. Differentially expressed RNAs and microRNAs between low and high NEDD4L expression groups.

Additional file 4: Table S3. Venn results of microRNAs targeting NEDD4L.

\section{Acknowledgements}

None.

\section{Authors' contributions}

$\mathrm{J}-\mathrm{dZ}$ and T-jZ conceived and designed the experiments; $\mathrm{M}-\mathrm{qC}$ performed the experiments; $\mathrm{L}-\mathrm{CZ}$ analyzed the data; $\mathrm{QY}$ collected the clinical data; J-dZ wrote the paper, All authors read and approved the final manuscript.

\section{Funding}

The work was supported by National Natural Science foundation of China (81900166), Zhenjiang Clinical Research Center of Hematology (SS2018009), Social Development Foundation of Zhenjiang (SH2020055), Medical Field of Zhenjiang "Jin Shan Ying Cai" Project, Scientific Research Foundation of Affiliated People's Hospital of Jiangsu University for PhD (KFB202002).

\section{Availability of data and materials}

All the data involved in this study had been included in the manuscript. The public data and the several datasets used and/or analyzed during the current study are available from the corresponding author on reasonable request.

\section{Declarations}

\section{Ethical approval and consent to participate}

The present study approved by the Ethics Committee of the Affiliated People's Hospital of Jiangsu University. Written informed consents were obtained from all enrolled individuals prior to their participation.

\section{Consent for publication}

All the co-authors agreed to publish the final version of this manuscript.

\section{Competing interests}

The authors declare that they have no competing interests. 


\section{Author details}

${ }^{1}$ Department of Hematology, Affiliated People's Hospital of Jiangsu University, 8 Dianli Rd., Zhenjiang 212002, Jiangsu, People's Republic of China. ${ }^{2}$ Laboratory Center, Affiliated People's Hospital of Jiangsu University, Zhenjiang, Jiangsu, People's Republic of China. ${ }^{3}$ Medical Laboratory, Qidong People's Hospital, Qidong, Jiangsu, People's Republic of China. ${ }^{4}$ Department of Oncology, Affiliated People's Hospital of Jiangsu University, 8 Dianli Rd., Zhenjiang 212002, Jiangsu, People's Republic of China.

Received: 26 July 2021 Accepted: 10 November 2021

Published online: 22 November 2021

\section{References}

1. Döhner H, Weisdorf DJ, Bloomfield CD. Acute myeloid leukemia. N Engl J Med. 2015;373(12):1136-52.

2. Charrot S, Armes H, Rio-Machin A, Fitzgibbon J. AML through the prism of molecular genetics. Br J Haematol. 2020;188(1):49-62.

3. Döhner H, Estey E, Grimwade D, Amadori S, Appelbaum FR, Büchner T, Dombret H, Ebert BL, Fenaux P, Larson RA, Levine RL, Lo-Coco F, Naoe T, Niederwieser D, Ossenkoppele GJ, Sanz M, Sierra J, Tallman MS, Tien HF, Wei AH, Löwenberg B, Bloomfield CD. Diagnosis and management of AML in adults: 2017 ELN recommendations from an international expert panel. Blood. 2017;129(4):424-47.

4. Wouters BJ, Löwenberg B, Delwel R. A decade of genome-wide gene expression profiling in acute myeloid leukemia: flashback and prospects. Blood. 2009;113(2):291-8.

5. Damm F, Heuser M, Morgan M, Wagner K, Görlich K, Grosshennig A, Hamwi I, Thol F, Surdziel E, Fiedler W, Lübbert M, Kanz L, Reuter C, Heil G, Delwel R, Löwenberg B, Valk PJ, Krauter J, Ganser A. Integrative prognostic risk score in acute myeloid leukemia with normal karyotype. Blood. 2011;117(17):4561-8.

6. Wang ZW, Hu X, Ye M, Lin M, Chu M, Shen X. NEDD4 E3 ligase: functions and mechanism in human cancer. Semin Cancer Biol. 2020;67(Pt 2):92-101.

7. Manning JA, Kumar S. Physiological functions of Nedd4-2: lessons from knockout mouse models. Trends Biochem Sci. 2018;43(8):635-47.

8. Goel P, Manning JA, Kumar S. NEDD4-2 (NEDD4L): the ubiquitin ligase for multiple membrane proteins. Gene. 2015;557(1):1-10.

9. Yang S, Tang D, Zhao YC, Liu H, Luo S, Stinchcombe TE, Glass C, Su L, Shen S, Christiani DC, Wang Q, Wei Q. Novel genetic variants in KIF16B and NEDD4L in the endosome-related genes are associated with nonsmall cell lung cancer survival. Int J Cancer. 2020;147(2):392-403.

10. Wang X, Duan J, Fu W, Yin Z, Sheng J, Lei Z, Wang H. Decreased expression of NEDD4L contributes to NSCLC progression and metastasis. Biochem Biophys Res Commun. 2019;513(2):398-404.

11. Jiang $X$, Zhang S, Yin Z, Sheng Y, Yan Q, Sun R, Lu M, Zhang Z, Li Y. The correlation between NEDD $4 \mathrm{~L}$ and HIF-1alpha levels as a gastric cancer prognostic marker. Int J Med Sci. 2019;16(11):1517-24.

12. Zhao F, Gong X, Liu A, Lv X, Hu B, Zhang H. Downregulation of NEDD4L predicts poor prognosis, promotes tumor growth and inhibits MAPK/ERK signal pathway in hepatocellular carcinoma. Biochem Biophys Res Commun. 2018;495(1):1136-43.

13. Yang Q, Zhao J, Cui M, Gi S, Wang W, Han X. NEDD4L expression is decreased in ovarian epithelial cancer tissues compared to ovarian noncancer tissue. J Obstet Gynaecol Res. 2015;41(12):1959-64.

14. Sakashita H, Inoue H, Akamine S, Ishida T, Inase N, Shirao K, Mori M, Mimori K. Identification of the NEDD4L gene as a prognostic marker by integrated microarray analysis of copy number and gene expression profiling in non-small cell lung cancer. Ann Surg Oncol. 2013;20(Suppl 3):S590-8.

15. He S, Deng J, Li G, Wang B, Cao Y, Tu Y. Down-regulation of NEDD4L is associated with the aggressive progression and worse prognosis of malignant glioma. Jpn J Clin Oncol. 2012;42(3):196-201.

16. Gao C, Pang L, Ren C, Ma T. Decreased expression of NEDD4L correlates with poor prognosis in gastric cancer patient. Med Oncol. 2012;29(3):1733-8.

17. Barretina J, Caponigro G, Stransky N, Venkatesan K, Margolin AA, Kim S, Wilson CJ, Lehár J, Kryukov GV, Sonkin D, Reddy A, Liu M, Murray L, Berger MF, Monahan JE, Morais P, Meltzer J, Korejwa A, Jané-Valbuena J, Mapa FA,
Thibault J, Bric-Furlong E, Raman P, Shipway A, Engels IH, Cheng J, Yu GK, Yu J, Aspesi P Jr, de Silva M, Jagtap K, Jones MD, Wang L, Hatton C, Palescandolo E, Gupta S, Mahan S, Sougnez C, Onofrio RC, Liefeld T, MacConail L, Winckler W, Reich M, Li N, Mesirov JP, Gabriel SB, Getz G, Ardlie K, Chan V, Myer VE, Weber BL, Porter J, Warmuth M, Finan P, Harris JL, Meyerson M, Golub TR, Morrissey MP, Sellers WR, Schlegel R, Garraway LA. The cancer cell line encyclopedia enables predictive modelling of anticancer drug sensitivity. Nature. 2012;483(7391):603-7.

18. Uhlén M, Fagerberg L, Hallström BM, Lindskog C, Oksvold P, Mardinoglu A, Sivertsson Å, Kampf C, Sjöstedt E, Asplund A, Olsson I, Edlund K, Lundberg E, Navani S, Szigyarto CA, Odeberg J, Djureinovic D, Takanen JO, Hober S, Alm T, Edqvist PH, Berling H, Tegel H, Mulder J, Rockberg J, Nilsson P, Schwenk JM, Hamsten M, von Feilitzen K, Forsberg M, Persson L, Johansson F, Zwahlen M, von Heijne G, Nielsen J, Pontén F. Proteomics. Tissue-based map of the human proteome. Science. 2015;347(6220):1260419.

19. Tang Z, Li C, Kang B, Gao G, Li C, Zhang Z. GEPIA: a web server for cancer and normal gene expression profiling and interactive analyses. Nucleic Acids Res. 2017;45(W1):W98-102.

20. Bagger FO, Kinalis S, Rapin N. BloodSpot: a database of healthy and malignant haematopoiesis updated with purified and single cell mRNA sequencing profiles. Nucleic Acids Res. 2019;47(D1):D881-5.

21. Cancer Genome Atlas Research Network, Ley TJ, Miller C, Ding L, Raphael BJ, Mungall AJ, Robertson A, Hoadley K, Triche TJ Jr, Laird PW, Baty JD, Fulton LL, Fulton R, Heath SE, Kalicki-Veizer J, Kandoth C, KIco JM, Koboldt DC, Kanchi KL, Kulkarni S, Lamprecht TL, Larson DE, Lin L, Lu C, McLellan MD, McMichael JF, Payton J, Schmidt H, Spencer DH, Tomasson MH, Wallis JW, Wartman LD, Watson MA, Welch J, Wendl MC, Ally A, Balasundaram M, Birol I, Butterfield Y, Chiu R, Chu A, Chuah E, Chun HJ, Corbett R, Dhalla N, Guin R, He A, Hirst C, Hirst M, Holt RA, Jones S, Karsan A, Lee D, Li HI, Marra MA, Mayo M, Moore RA, Mungall K, Parker J, Pleasance E, Plettner P, Schein J, Stoll D, Swanson L, Tam A, Thiessen N, Varhol R, Wye N, Zhao Y, Gabriel S, Getz G, Sougnez C, Zou L, Leiserson MD, Vandin F, Wu HT, Applebaum F, Baylin SB, Akbani R, Broom BM, Chen K, Motter TC, Nguyen K, Weinstein JN, Zhang N, Ferguson ML, Adams C, Black A, Bowen J, Gastier-Foster J, Grossman T, Lichtenberg T, Wise L, Davidsen T, Demchok JA, Shaw KR, Sheth M, Sofia HJ, Yang L, Downing JR, Eley G. Genomic and epigenomic landscapes of adult de novo acute myeloid leukemia. $\mathrm{N}$ Engl J Med. 2013;368(22):2059-74.

22. Metzeler KH, Hummel M, Bloomfield CD, Spiekermann K, Braess J, Sauerland MC, Heinecke A, Radmacher M, Marcucci G, Whitman SP, Maharry K, Paschka P, Larson RA, Berdel WE, Büchner T, Wörmann B, Mansmann U, Hiddemann W, Bohlander SK, Buske C, Cancer and Leukemia Group B; German AML Cooperative Group. An 86-probe-set gene-expression signature predicts survival in cytogenetically normal acute myeloid leukemia. Blood. 2008;112:4193-201.

23. Kassambara A, Rème T, Jourdan M, Fest T, Hose D, Tarte K, Klein B. GenomicScape: an easy-to-use web tool for gene expression data analysis. Application to investigate the molecular events in the differentiation of $B$ cells into plasma cells. PLoS Comput Biol. 2015;11:e1004077.

24. Zhang TJ, Xu ZJ, Gu Y, Wen XM, Ma JC, Zhang W, Deng ZQ, Leng JY, Qian J, Lin J, Zhou JD. Identification and validation of prognosis-related DLX5 methylation as an epigenetic driver in myeloid neoplasms. Clin Transl Med. 2020;10(2):e29.

25. Zhou JD, Zhang TJ, Xu ZJ, Deng ZQ, Gu Y, Ma JC, Wen XM, Leng JY, Lin J, Chen SN, Qian J. Genome-wide methylation sequencing identifies progression-related epigenetic drivers in myelodysplastic syndromes. Cell Death Dis. 2020;11(11):997.

26. Zhang TJ, Xu ZJ, Gu Y, Ma JC, Wen XM, Zhang W, Deng ZQ, Qian J, Lin J, Zhou JD. Identification and validation of obesity-related gene LEP methylation as a prognostic indicator in patients with acute myeloid leukemia. Clin Epigenetics. 2021;13(1):16.

27. Zhang TJ, Zhou JD, Zhang W, Lin J, Ma JC, Wen XM, Yuan Q, Li XX, Xu ZJ, Qian J. H19 overexpression promotes leukemogenesis and predicts unfavorable prognosis in acute myeloid leukemia. Clin Epigenetics. 2018;10:47.

28. Dong H, Zhu L, Sun J, Zhang Y, Cui Q, Wu L, Chen S, Lu J. Pan-cancer analysis of NEDD $4 L$ and its tumor suppressor effects in clear cell renal cell carcinoma. J Cancer. 2021;12(20):6242-53.

29. Zhang TJ, Zhou JD, Ma JC, Deng ZQ, Qian Z, Yao DM, Yang J, Li XX, Lin J, Qian J. CDH1 (E-cadherin) expression independently affects clinical 
outcome in acute myeloid leukemia with normal cytogenetics. Clin Chem Lab Med. 2017;55(1):123-31.

30. Chu MQ, Zhang TJ, Xu ZJ, Gu Y, Ma JC, Zhang W, Wen XM, Lin J, Qian J, Zhou JD. EZH2 dysregulation: Potential biomarkers predicting prognosis and guiding treatment choice in acute myeloid leukaemia. J Cell Mol Med. 2020;24(2):1640-9.

31. Wang Z, Hong Z, Gao F, Feng W. Upregulation of microRNA-375 is associated with poor prognosis in pediatric acute myeloid leukemia. Mol Cell Biochem. 2013:383(1-2):59-65.

32. Bryant A, Palma CA, Jayaswal V, Yang YW, Lutherborrow M, Ma DD. miR-10a is aberrantly overexpressed in Nucleophosmin1 mutated acute myeloid leukaemia and its suppression induces cell death. Mol Cancer. 2012;11:8.

33. Vu TT, Stölzel F, Wang KW, Röllig C, Tursky ML, Molloy TJ, Ma DD. miR-10a as a therapeutic target and predictive biomarker for MDM2 inhibition in acute myeloid leukemia. Leukemia. 2020. https://doi.org/10.1038/ s41375-020-01095-Z.

34. Zhang TJ, Guo H, Zhou JD, Li XX, Zhang W, Ma JC, Wen XM, Yao XY, Lin J, Qian J. Bone marrow miR-10a overexpression is associated with genetic events but not affects clinical outcome in acute myeloid leukemia. Pathol Res Pract. 2018;214(1):169-73.

35. Sun Y, Wang H, Luo C. MiR-100 regulates cell viability and apoptosis by targeting ATM in pediatric acute myeloid leukemia. Biochem Biophys Res Commun. 2020;522(4):855-61.

36. Bai J, Guo A, Hong Z, Kuai W. Upregulation of microRNA-100 predicts poor prognosis in patients with pediatric acute myeloid leukemia. Onco Targets Ther. 2012;5:213-9.
37. Hua S, Feng T, Yin L, Wang Q, Shao X. NEDD9 overexpression: prognostic and guidance value in acute myeloid leukaemia. J Cell Mol Med. 2021;25(19):9331-9.

38. Zeng Y, Feng Z, Liao Y, Yang M, Bai Y, He Z. Diminution of microRNA-98 alleviates renal fibrosis in diabetic nephropathy by elevating NEDD4L and inactivating TGF- $\beta /$ Smad2/3 pathway. Cell Cycle. 2020;19(24):3406-18.

39. Tian RH, Guo KM, Han GH, Bai Y. Downregulation of MicroRNA-494 inhibits the TGF-beta1/Smads signaling pathway and prevents the development of hypospadias through upregulating NEDD4L. Exp Mol Pathol. 2020;115:104452

40. Chen KC, Chen PH, Ho KH, Shih CM, Chou CM, Cheng CH, Lee CC. IGF-1-enhanced miR-513a-5p signaling desensitizes glioma cells to temozolomide by targeting the NEDD4L-inhibited Wnt/beta-catenin pathway. PLOS ONE. 2019;14(12):e0225913.

41. Guarnieri AL, Towers CG, Drasin DJ, Oliphant MUJ, Andrysik Z, Hotz TJ, Vartuli RL, Linklater ES, Pandey A, Khanal S, Espinosa JM, Ford HL. The miR-106b-25 cluster mediates breast tumor initiation through activation of NOTCH1 via direct repression of NEDD4L. Oncogene. 2018;37(28):3879-93.

42. Zhu JY, Heidersbach A, Kathiriya IS, Garay BI, Ivey KN, Srivastava D, Han Z, King IN. The E3 ubiquitin ligase Nedd4/NEDD4L is directly regulated by microRNA 1. Development. 2017;144(5):866-75.

\section{Publisher's Note}

Springer Nature remains neutral with regard to jurisdictional claims in published maps and institutional affiliations.
Ready to submit your research? Choose BMC and benefit from:

- fast, convenient online submission

- thorough peer review by experienced researchers in your field

- rapid publication on acceptance

- support for research data, including large and complex data types

- gold Open Access which fosters wider collaboration and increased citations

- maximum visibility for your research: over 100M website views per year

At BMC, research is always in progress.

Learn more biomedcentral.com/submissions 